\title{
On the DDT theorem
}

\author{
by
}

\section{G. Bareikis and E. Manstavičius (Vilnius)}

Dedicated to our teacher Professor Jonas Kubilius on his 85th birthday

1. Introduction. We denote the sets of natural, real, complex, and prime numbers by $\mathbb{N}, \mathbb{R}, \mathbb{C}$, and $\mathbb{P}$ respectively. In what follows we assume that $p \in \mathbb{P}, d, l, m, n \in \mathbb{N}$, and $s=\sigma+i \tau \in \mathbb{C}$. The letters $c$ and $C$ with or without subscripts denote constants. Either of the notation $f=$ $\mathrm{O}(g)$ or $f \ll g$ will mean that $|f| \leq C|g|$ for some positive constant $C$, which may be absolute or depend upon various parameters. In such cases we sometimes indicate that by a subscript. Throughout the paper $x \geq 3$ will be the sequence parameter and in the asymptotic relations it is assumed that $x \rightarrow \infty$.

Let $\tau(m, v)$ be the number of natural divisors of $m$ which do not exceed $v \leq m$, and $\tau(m)=\tau(m, m)$. In 1979 J.-M. Deshouillers, F. Dress, and G. Tenenbaum [2] (see also [10, Section II.6.2]) obtained the following result.

Theorem DDT. Uniformly in $u \in[0,1]$,

$$
\frac{1}{x} \sum_{m \leq x} \frac{\tau\left(m, m^{u}\right)}{\tau(m)}=\frac{2}{\pi} \arcsin \sqrt{u}+\mathrm{O}\left(\frac{1}{\sqrt{\log x}}\right) .
$$

This asymptotic formula was announced already in [3], where some argument relating it to the well known arcsine law in the probabilistic invariance principle was presented. In our opinion, that argument was not very convincing. To support that, we observe that the arcsine law in the classical probability theory is in some sense universal while (1) fails to hold if one replaces $\tau(m)$ by another multiplicative number-theoretic function whose values on prime numbers differ from two fairly often. This raises a desire to search for another limiting relation generalizing (1). Such an attempt was made by the second author who in [6, Section 8$]$ announced a result

2000 Mathematics Subject Classification: 11K65, 11N60.

Key words and phrases: multiplicative function, mean value, natural divisor, beta distribution. 
showing that, instead of the arcsine function, a new limit belonging to the class of beta distributions can appear. This phenomenon was also reported by the first author in the semigroup of polynomials over a finite field [1]. In the present note, we generalize the DDT theorem and slightly sharpen the remainder term.

Relation (1) arouses interest from the point of view of arithmetically defined random processes. First, observe that (1) will remain true if $\tau\left(m, m^{u}\right)$ is replaced by $\tau\left(m, x^{u}\right)$. So, the limit in (1) can be understood as the asymptotic expectation of the sequence of stochastic processes if a number $m \leq x$ is taken with probability $\nu_{x}(\{m\})=1 /[x]$. Later results obtained in [9] dealt with the asymptotic distribution of increments

$$
\left(\tau\left(m, m^{v}\right)-\tau\left(m, m^{u}\right)\right) / \tau(m),
$$

if $0 \leq u \leq v \leq 1$. Having all this in mind, the second author [5] asked whether the distributions of the processes $\tau\left(m, x^{u}\right) / \tau(m)$ under the measure $\nu_{x}$ weakly converge in the Skorokhod space $\mathbb{D}[0,1]$. This hypothesis together with some generalization was proved in [7]. To formulate the result, we recall some definition.

The space $\mathbb{D}[0,1]$ consists of the real-valued functions on $[0,1]$ which are right-continuous and have left-hand limits. It is assumed that the Skorokhod metric is introduced in it. Write $\mathcal{D}$ for the Borel $\sigma$-algebra in $\mathbb{D}[0,1]$. For a multiplicative function $f: \mathbb{N} \rightarrow \mathbb{R}^{+}$, we define

$$
T(m, v)=\sum_{d \mid m, d \leq v} f(d), \quad X_{x}(m, u)=\frac{T\left(m, x^{u}\right)}{T(m)}, \quad T(m)=T(m, m),
$$

where $u, v \in[0,1]$. Let $\nu_{x} \circ X_{x}^{-1}$ be the induced measure on $\mathcal{D}$.

Theorem MT. If $f(p)=\varkappa>0$ for each $p \in \mathbb{P}$ and $f\left(p^{k}\right) \geq 0$ for all $p \in \mathbb{P}$ and $k \geq 2$, then $\nu_{x} \circ X_{x}^{-1}$ weakly converges to a limit measure on $\mathcal{D}$.

In the Addendum [11], G. Tenenbaum generalized this theorem and showed, in addition, that the limit measure is concentrated on the subspace of continuous functions. A simple example which does not satisfy the conditions of Theorem MT but is covered by the result of [11] is defined via the multiplicative function with $f(p)=1+(-1)^{(p-1) / 2}$ for all odd $p \in \mathbb{P}$. What is the asymptotic mean value of this particular process? In the present paper, we give a more general answer.

2. Results. Let $f: \mathbb{N} \rightarrow \mathbb{R}^{+}$be a multiplicative function. Throughout the paper we assume that, for some constant $C>0$, it satisfies $f\left(p^{l}\right) \leq C$ for all $p \in \mathbb{P}$ and $l \in \mathbb{N}$. We say that $f$ belongs to the class $\mathcal{M}(\alpha), 0<\alpha<1$, 
if the function defined by the series

$$
\sum_{p}\left(\frac{1}{1+f(p)}-\alpha\right) \frac{1}{p^{s}}, \quad \sigma>1,
$$

for some $c>0$ and $0<\delta<1$, has an analytic continuation $P(s)$ into the region

$$
\sigma \geq \sigma(t):=1-\frac{c}{\log (|t|+2)}, \quad t \in \mathbb{R}
$$

where $P(s)$ is holomorphic and $|P(s)| \leq(1-\delta) \log (|t|+2)$.

The well known formula

$$
\sum_{p} \frac{1}{p^{s}}=\log \zeta(s)+H(s)
$$

where $H(s)$ is analytic in $\sigma>1 / 2$, gives an analytic continuation of the sum on the left-hand side into the region $\sigma \geq \sigma(t)$, where it is a holomorphic function, say, $L(s)$. Moreover, $L(s) \ll \log \log (2+|t|)$ there. Thus, if $f \in$ $\mathcal{M}(\alpha)$, then, for $\beta:=1-\alpha$, the function

$$
\sum_{p}\left(\frac{f(p)}{1+f(p)}-\beta\right) \frac{1}{p^{s}}
$$

again has the same properties. This observation will be repeatedly exploited in what follows.

Throughout the paper, the dependence of the appearing remainders on the constants $\alpha, \delta, c$ and $C$ involved in the definition of the class $\mathcal{M}(\alpha)$ is allowed. Using (2), we define

$$
S_{x}(u)=\frac{1}{x} \sum_{m \leq x} \frac{T\left(m, m^{u}\right)}{T(m)}
$$

and $S_{x}(u, v)=S_{x}(v)-S_{x}(u)$ where $0 \leq u \leq v \leq 1$. Recall that the distribution function of a beta law has the following expression:

$$
B(u ; a, b)=\frac{1}{\Gamma(a) \Gamma(b)} \int_{0}^{u} \frac{d v}{v^{a}(1-v)^{b}}, \quad 0 \leq u \leq 1,
$$

where $0<a=1-b<1$ and $\Gamma(z)$ denotes the Euler gamma-function. Set $B(u, v ; a, b)=B(v ; a, b)-B(u ; a, b)$ for $0 \leq u \leq v \leq 1$ and $a \wedge b=\min \{a, b\}$.

THEOREM 1. If $f \in \mathcal{M}(\alpha)$, then

$$
S_{x}(u)-B(u ; \alpha, \beta) \ll \frac{1}{\log ^{\alpha} x}+\frac{1}{\log ^{\beta} x}
$$

uniformly in $0 \leq u \leq 1$.

This result follows from the next estimate which is sharper in the central zone. 
Theorem 2. If $f \in \mathcal{M}(\alpha)$ and $0 \leq u \leq v \leq 1$, then

$$
\begin{aligned}
S_{x}(u, v)-B(u, v ; \alpha, \beta) \ll & \frac{1}{\log ^{\beta} x}\left(1 \wedge \frac{1}{(u \log x)^{\alpha}}\right) \\
& +\frac{1}{\log ^{\alpha} x}\left(1 \wedge \frac{1}{((1-u) \log x)^{\beta}}\right) .
\end{aligned}
$$

For the functions discussed in Theorem MT, one can take $\alpha=(1+\varkappa)^{-1}$.

Generalizing the above mentioned example we have the following result.

Corollary. Fix $m \in \mathbb{N}$ and let $a_{l} \in \mathbb{R}^{+}$(not all zero) for $1 \leq l<m$ and $(l, m)=1$. If $f: \mathbb{N} \rightarrow \mathbb{R}^{+}$is the multiplicative function defined by $f(p)=a_{l}$ for $p \equiv l(\bmod m)$, where $1 \leq l<m$ and $(l, m)=1, f(p)=0$ otherwise, and $0 \leq f\left(p^{k}\right) \leq C_{1}$ for $p \in \mathbb{P}$ and $k \geq 2$, then the assertion of Theorem 2 holds with

$$
1-\beta:=\alpha:=\frac{1}{\varphi(m)} \sum_{\substack{l<m \\(l, m)=1}} \frac{1}{1+a_{l}} .
$$

In the proofs, we apply the analytic approach proposed in [2].

3. Auxiliary lemmas. As usual, let $\zeta(s)$ be the Riemann zeta function. First, we present two classical lemmas.

Lemma 1 ([10, Section II.5.3]). Let $z \in \mathbb{C}, M>0,0 \leq \varrho<1$, and $F(s):=\sum_{n=1}^{\infty} a_{n} / n^{s}, a_{n} \geq 0$, be such that the function $G_{z}(s):=F(s) \zeta^{-z}(s)$ can be continued as a holomorphic function for $\sigma \geq 1-c_{1} / \log (|t|+2), t \in \mathbb{R}$, and in this domain satisfies the bound

$$
\left|G_{z}(s)\right| \leq M(1+|\tau|)^{\varrho} .
$$

Then, for $A>0$ and $|z| \leq A$, we have

$$
\sum_{n \leq x} a_{n}=\frac{x}{\log ^{1-z} x}\left(\frac{G_{z}(1)}{\Gamma(z)}+\mathrm{O}\left(\frac{M}{\log x}\right)\right),
$$

where the implicit constant of the remainder depends at most on $c_{1}, \varrho$, and $A$.

Lemma 2 ([4]). Let $g$ be a multiplicative function with $0 \leq g\left(p^{l}\right) \leq C_{1}$ for all prime numbers $p$ and $l \in \mathbb{N}$. Then

$$
\begin{aligned}
& \sum_{m \leq x} g(m) \ll_{C_{1}} \frac{x}{\log x} \exp \left\{\sum_{p \leq x} \frac{g(p)}{p}\right\}, \\
& \sum_{m \leq x} \frac{g(m)}{m} \ll_{C_{1}} \exp \left\{\sum_{p \leq x} \frac{g(p)}{p}\right\} .
\end{aligned}
$$


Let $T(m)$ be as defined in Section 1. Then

$$
T\left(p^{k}\right)=1+f(p)+\cdots+f\left(p^{k}\right) .
$$

For the multiplicative function $h(m)=f(m)$ or $h(m)=I(m): \equiv 1$, we define

$$
Q_{x}(d ; h)=\sum_{n \leq x} \frac{h(n)}{T(n d)}, \quad F(a ; h)=\prod_{p}\left(1-\frac{1}{p}\right)^{a} \sum_{i=0}^{\infty} \frac{h\left(p^{i}\right)}{T\left(p^{i}\right) p^{i}}
$$

where $a>0$. Similarly,

$$
g(d ; h):=\prod_{p^{l} \| d} g\left(p^{l} ; h\right), \quad g\left(p^{l} ; h\right)=\left(\sum_{i=0}^{\infty} \frac{h\left(p^{i}\right)}{T\left(p^{i+l}\right) p^{i}}\right) /\left(\sum_{i=0}^{\infty} \frac{h\left(p^{i}\right)}{T\left(p^{i}\right) p^{i}}\right),
$$

$g(d):=g(d ; f)$, and $g^{*}(d):=g(d ; I)$. Further, set

$$
r(d)=\prod_{p \mid d} \frac{1}{1+f(p)}\left(1+\frac{C_{2}}{p^{\sigma_{0}}}\right),
$$

where $C_{2}>0$ and $0<\sigma_{0}<1$ are arbitrary.

Lemma 3. If $f \in \mathcal{M}(\alpha)$, then, for all $d \in \mathbb{N}$,

$$
\begin{aligned}
& \frac{1}{x} Q_{x}(d ; f)=\frac{1}{\Gamma(\beta) \log ^{\alpha} x}\left(F(\beta ; f) g(d)+\mathrm{O}\left(\frac{r(d)}{\log x}\right)\right), \\
& \frac{1}{x} \sum_{d \leq x} g(d)=\frac{1}{\Gamma(\alpha) F(\beta ; f) \log ^{\beta} x}\left(1+\mathrm{O}\left(\frac{1}{\log x}\right)\right) .
\end{aligned}
$$

Moreover,

$$
\frac{1}{x} \sum_{d \leq x} r(d) \ll \log ^{-\beta} x, \quad \frac{1}{x} \sum_{d \leq x} f(d) r(d) \ll \log ^{-\alpha} x .
$$

Proof. Introduce the generating series

$$
\begin{aligned}
\phi(s ; d, f) & =\sum_{n \geq 1} \frac{f(n)}{T(n d) n^{s}} \\
& =\prod_{p^{k} \| d}\left(\sum_{i=0}^{\infty} \frac{f\left(p^{i}\right)}{T\left(p^{i+k}\right) p^{i s}}\right)\left(\sum_{i=0}^{\infty} \frac{f\left(p^{i}\right)}{T\left(p^{i}\right) p^{i s}}\right)^{-1} \cdot \prod_{p} \sum_{i=0}^{\infty} \frac{f\left(p^{i}\right)}{T\left(p^{i}\right) p^{i s}} \\
& =: g(s ; d, f) \phi(s ; 1, f) .
\end{aligned}
$$

Since $f\left(p^{i}\right) / T\left(p^{i+k}\right) \leq f\left(p^{i}\right) /\left(1+f\left(p^{i}\right)\right) \leq C /(1+C)<1$ for each $k \geq 0$ and $i \geq 1$, the last expansion is valid for $\sigma>1$. 
We now examine the factors. First we observe that

$$
\begin{aligned}
\left|v_{p}(s ; f)\right| & :=\left|1+\sum_{i=1}^{\infty} \frac{f\left(p^{i}\right)}{T\left(p^{i}\right) p^{i s}}\right| \geq 1-\frac{C}{(1+C)\left(p^{\sigma}-1\right)} \\
& \geq 1-\left(\frac{C}{1+C}\right)^{1 / 2}>0
\end{aligned}
$$

for every $p \in \mathbb{P}$ provided that

$$
\sigma \geq \sigma_{0}=\max \left\{3 / 4, \log _{2}\left(1+(C /(1+C))^{1 / 2}\right)\right\} .
$$

Hence in the same region, if $k \geq 1$,

$$
\begin{aligned}
\left|g\left(s ; p^{k}, f\right)\right| & =\left|\sum_{i=0}^{\infty} \frac{f\left(p^{i}\right)}{T\left(p^{i+k}\right) p^{i s}}\right|\left|\sum_{i=0}^{\infty} \frac{f\left(p^{i}\right)}{T\left(p^{i}\right) p^{i s}}\right|^{-1} \\
& \leq \frac{1}{1+f(p)}\left(1+\frac{C}{p^{\sigma_{0}}}+\mathrm{O}\left(\frac{1}{p^{2 \sigma_{0}}}\right)\right)\left(1-\frac{C}{(1+C)\left(p^{\sigma_{0}}-1\right)}\right)^{-1} \\
& \leq \frac{1}{1+f(p)}\left(1+\left(C+\frac{C}{1+C}\right) \frac{1}{p^{\sigma_{0}}}+\mathrm{O}\left(\frac{1}{p^{2 \sigma_{0}}}\right)\right) .
\end{aligned}
$$

Consequently,

$$
\begin{aligned}
|g(s ; d, f)| & =\prod_{p^{k} \| d}\left|g\left(s ; p^{k}, f\right)\right| \\
& \ll \prod_{p \mid d} \frac{1}{1+f(p)}\left(1+\left(C+\frac{C}{1+C}\right) \frac{1}{p^{\sigma_{0}}}\right)=r(d)
\end{aligned}
$$

if $\sigma \geq \sigma_{0}$.

Examine the function

$$
\begin{aligned}
G(s) & :=\phi(s ; 1, f) \zeta^{-\beta}(s)=\prod_{p}\left(1+\frac{f(p)}{T(p) p^{s}}+\sum_{k=2}^{\infty} \frac{f\left(p^{k}\right)}{T\left(p^{k}\right) p^{k s}}\right)\left(1-\frac{1}{p^{s}}\right)^{\beta} \\
& =\exp \left\{\sum_{p}\left(\frac{f(p)}{1+f(p)}-\beta\right) \frac{1}{p^{s}}\right\} H(s) .
\end{aligned}
$$

Here $H(s)$ is some product over primes. In the routine way, taking logarithms, which is allowed by (8), we verify that the function $H(s)$ is analytic and bounded for $\sigma \geq \sigma_{0}$. Dealing with the sum under the exponent, we exploit the definition of the class $\mathcal{M}(\alpha)$. Thus, $G(s)$ has an analytic continuation into the region $\sigma \geq \sigma(t)$, where it is holomorphic and $G(s) \ll(2+|t|)^{1-\delta}$ for some $0<\delta<1$. Together with (7) and (8) this also implies an analytic continuation of $\phi(s ; d, f) \zeta^{-\beta}(s)$ and the estimate

$$
\phi(s ; d, f) \zeta^{-\beta}(s)=g(s ; d, f) G(s) \ll r(d)(2+|t|)^{1-\delta}
$$


for $\sigma \geq \sigma(t)$. Since $g(1 ; d, f)=g(d)$, by Lemma 1 we obtain (4) with $F(\beta ; f)=G(1)$.

To derive the asymptotic formula (5), we will apply Lemma 1 for

$$
G_{\alpha}(s)=\zeta(s)^{-\alpha} \sum_{n=1}^{\infty} \frac{g(n)}{n^{s}}=\prod_{p}\left(1-\frac{1}{p^{s}}\right)^{\alpha}\left(1+\sum_{l=1}^{\infty} \frac{g\left(p^{l}\right)}{p^{l s}}\right) .
$$

By definition of $g\left(p^{k}\right)$, we obtain

$$
G_{\alpha}(s)=\prod_{p}\left(1+\left(\frac{1}{1+f(p)}-\alpha\right) \frac{1}{p^{s}}+\frac{u_{p}(s)}{p^{2 s}}\right),
$$

where $\left|u_{p}(s)\right| \leq C_{3}$ uniformly in $\sigma \geq 3 / 4$ and $p \in \mathbb{P}$. Separating a finite number of factors, with $p \leq p_{0}$, where $p_{0}$ is sufficiently large, we can ensure that the remaining factors (for $p>p_{0}$ ) do not vanish for $\sigma>1$. So expanding their logarithms we obtain the representation

$$
G_{\alpha}(s)=\exp \left\{\sum_{p}\left(\frac{1}{1+f(p)}-\alpha\right) \frac{1}{p^{s}}\right\} H_{1}(s),
$$

where $H_{1}(s)$ is an analytic and bounded function in $\sigma \geq 3 / 4$. Again, since $f \in \mathcal{M}(\alpha)$, having an analytic expansion of the series under the exponent and the appropriate estimate, we can apply Lemma 1 with $M=1$. This yields

$$
\sum_{d \leq x} g(d)=\frac{x}{\Gamma(\alpha) \ln ^{\beta} x}\left(G_{\alpha}(1)+\mathrm{O}\left(\frac{1}{\log x}\right)\right),
$$

where $G_{\alpha}(1)>0$.

It remains to show that $G_{\alpha}(1) F(\beta ; f)=1$, where $F(\beta ; f)$ has been defined in (3). If $x(p):=v_{p}(1 ; f)$, using $\alpha+\beta=1$, we obtain

$$
\begin{aligned}
G_{\alpha}(1) F(\beta ; f) & =\prod_{p}\left(1-\frac{1}{p}\right)\left(1+\frac{1}{x(p)} \sum_{l=1}^{\infty} \frac{1}{p^{l}} \sum_{i=0}^{\infty} \frac{f\left(p^{i}\right)}{T\left(p^{l+i}\right) p^{i}}\right) x(p) \\
& =\prod_{p}\left(1-\frac{1}{p}\right)\left(x(p)+\sum_{i=0}^{\infty} f\left(p^{i}\right) \sum_{j=i+1}^{\infty} \frac{1}{T\left(p^{j}\right) p^{j}}\right) \\
& =\prod_{p}\left(1-\frac{1}{p}\right)\left(x(p)+\sum_{j=1}^{\infty} \frac{1}{p^{j} T\left(p^{j}\right)} \sum_{i=0}^{j-1} f\left(p^{i}\right)\right) \\
& =\prod_{p}\left(1-\frac{1}{p}\right)\left(x(p)+\sum_{j=1}^{\infty} \frac{1}{p^{j}}-(x(p)-1)\right)=1 .
\end{aligned}
$$

Thus, relation (5) is proved. 
Since $f \in \mathcal{M}(\alpha)$ implies

$$
\sum_{p \leq x} \frac{r(p)}{p}=\sum_{p \leq x} \frac{1}{p(1+f(p))}+\mathrm{O}(1)=\alpha \log \log x+\mathrm{O}(1),
$$

the first estimate in (6) is a corollary of Lemma 2. Similarly, we obtain the second one.

Lemma 3 is proved.

In a similar manner we prove the next lemma.

Lemma 4. If $f \in \mathcal{M}(\alpha)$, then, for all $d \in \mathbb{N}$,

$$
\frac{1}{x} Q_{x}(d ; I)=\frac{1}{\Gamma(\alpha) \log ^{\beta} x}\left(F(\alpha ; I) g^{*}(d)+\mathrm{O}\left(\frac{r(d)}{\log x}\right)\right) .
$$

Moreover,

$$
\frac{1}{x} \sum_{d \leq x} g^{*}(d) f(d)=\frac{1}{\Gamma(\beta) F(\alpha ; I) \log ^{\alpha} x}\left(1+\mathrm{O}\left(\frac{1}{\log x}\right)\right) .
$$

Proof. We now start with

$$
\begin{aligned}
\phi(s ; d, I) & =\sum_{n \geq 1} \frac{1}{T(n d) n^{s}} \\
& =\prod_{p^{k} \| d}\left(\sum_{i=0}^{\infty} \frac{1}{T\left(p^{i+k}\right) p^{i s}}\right)\left(\sum_{i=0}^{\infty} \frac{1}{T\left(p^{i}\right) p^{i s}}\right)^{-1} \cdot \prod_{p} \sum_{i=0}^{\infty} \frac{1}{T\left(p^{i}\right) p^{i s}} \\
& =g(s ; d, I) \phi(s ; 1, I) .
\end{aligned}
$$

For the factor appearing in the denominator,

$$
v_{p}(s ; I)=1+\sum_{i=1}^{\infty} \frac{1}{T\left(p^{i}\right) p^{i s}},
$$

as in (8), we have

$$
\left|v_{p}(s ; I)\right| \geq 1-\frac{1}{3^{\sigma}-1} \geq c_{2}>0
$$

for every $p \geq 3$ if $\sigma \geq 1-c_{3}$ with sufficiently small $c_{3}<1$. If $f\left(2^{k}\right) \equiv 0$ for $k \geq 1$, then $\left|v_{2}(s ; I)\right|=\left|1-2^{-s}\right|^{-1} \geq c_{4}>0$ in the same region. If $f\left(2^{k}\right)=c_{5}>0$ for some $k \geq 1$, then

$$
\left|v_{2}(s ; I)\right| \geq 1-\frac{1}{2^{\sigma}-1}+\frac{1}{2^{k \sigma}}\left(1-\frac{1}{1+c_{5}}\right)
$$

for $\sigma>0$. Hence $v_{2}(1, I) \geq c_{5}\left(1+c_{5}\right)^{-1} 2^{-k}>0$ which shows that the continuous function $\left|v_{2}(s, I)\right|$ will remain bounded from above by some positive constant if $\sigma \geq 1-c_{6}$ with sufficiently small $c_{6}$. In the following, let 
$\sigma_{0}=1-\min \left\{c_{3}, c_{6}\right\}$. Now, as in the proof of Lemma 3 , we derive the estimate

$$
|g(s ; d, I)| \ll \prod_{p \mid d} \frac{1}{1+f(p)}\left(1+\frac{2}{p^{\sigma_{0}}}\right)=r(d)
$$

if $\sigma \geq \sigma_{0}$.

Further, expanding the logarithms of non-vanishing factors, we obtain the representation

$$
\phi(s ; 1, I)=\zeta^{\alpha}(s) \exp \left\{\sum_{p}\left(\frac{1}{1+f(p)}-\alpha\right) \frac{1}{p^{s}}\right\} H_{2}(s),
$$

where $\mathrm{H}_{2}(s)$ is an analytic and bounded function in $\sigma \geq 3 / 4$. Again, by Theorem 1, having an analytic expansion of the series under the exponent and the appropriate estimate, we can apply Lemma 1 with $M=r(d)$. So, since $g(1 ; d, I)=g^{*}(d)$, we obtain (9).

Finally, we examine

$$
W_{\beta}(s):=\zeta(s)^{-\beta} \sum_{n=1}^{\infty} \frac{g^{*}(n) f(n)}{n^{s}}, \quad \sigma>1 .
$$

By the definition we obtain $g^{*}(p) f(p)=f(p) / T(p)+\mathrm{O}(1 / p)$ and

$$
W_{\beta}(s)=\prod_{p}\left(1+\left(\frac{f(p)}{1+f(p)}-\beta\right) \frac{1}{p^{s}}+\frac{w_{p}(s)}{p^{2 s}}\right),
$$

where $w_{p}(s)$ are bounded for $\sigma \geq 3 / 4$. By the same argument as when dealing with $G_{\alpha}(s)$ earlier, we arrive at the formula

$$
W_{\beta}(s)=\exp \left\{\sum_{p}\left(\frac{f(p)}{1+f(p)}-\beta\right) \frac{1}{p^{s}}\right\} H_{3}(s),
$$

where $H_{3}(s)$ is analytic in the region $\sigma \geq 3 / 4$. Since $f \in \mathcal{M}(\alpha)$, applying Lemma 1 with $z=\beta$ and $M=1$, we obtain

$$
\sum_{k \leq x} g^{*}(k) f(k)=\frac{x}{\Gamma(\beta) \log ^{\alpha} x}\left(W_{\beta}(1)+\mathrm{O}\left(\frac{1}{\log x}\right)\right),
$$

where

$$
W_{\beta}(1)=\prod_{p}\left(1-\frac{1}{p}\right)^{\beta}\left(\sum_{i=0}^{\infty} \frac{f\left(p^{i}\right) g\left(p^{i}\right)}{p^{i}}\right)>0 .
$$

Repeating the similar argument above, we obtain $W_{\beta}(1) F(\alpha, 1)=1$. This and (11) yield the desired equality (10).

Lemma 4 is proved.

4. Proof of Theorem 2. If $u \leq 1 / \log x$ or $1-1 / \log x \leq v \leq 1$, the assertion of Theorem 2 follows from easy estimates of the tails of a beta 
distribution. Moreover, we can concentrate on the cases: (A) $1 / \log x \leq u \leq$ $1 / 2=v$ and (B) $u=1 / 2 \leq v \leq 1-1 / \log x$.

(A) For convenience, in the definition of $S_{x}(u)$ we replace $n^{u}$ by $x^{u}$. The error at this step is negligible. Indeed, checking monotonicity of the appropriate factors in sums, we have

$$
\begin{aligned}
R_{x}(u) & :=\frac{1}{x} \sum_{n \leq x} \frac{1}{T(n)} \sum_{\substack{d \mid n \\
n^{u}<d \leq x^{u}}} f(d) \\
& =\frac{1}{x} \sum_{d \leq x^{u}} f(d) \sum_{\substack{m \leq x / d \\
m<d^{(1-u) / u}}} \frac{1}{T(m d)} \\
& \ll \frac{1}{x}+\frac{u^{\beta}}{x} \sum_{3 \leq d \leq x^{u}} \frac{f(d) d^{(1-u) / u}}{\log ^{\beta} d}\left(g^{*}(d)+\mathrm{O}\left(\frac{u r(d)}{\log d}\right)\right) \\
& \ll \frac{1}{x^{u} \log ^{\beta} x} \sum_{d \leq x^{u}} f(d) g^{*}(d)+\frac{1}{x^{u} \log ^{1+\beta} x} \sum_{d \leq x^{u}} f(d) r(d)+\frac{1}{x} .
\end{aligned}
$$

Now, by Lemmas 3 and 4 , we obtain $R_{x}(u) \ll u^{-\alpha} \log ^{-1} x$ uniformly in $1 / \log x \leq u \leq 1 / 2$. Thus,

$$
S_{x}(u, 1 / 2)=\widehat{S}_{x}(1 / 2)-\widehat{S}_{x}(u)+\mathrm{O}\left(u^{-\alpha} \log ^{-1} x\right),
$$

where by Lemma 4 ,

$$
\begin{aligned}
\widehat{S}_{x}(u) & :=\frac{1}{x} \sum_{n \leq x} \frac{T\left(n, x^{u}\right)}{T(m)}=\frac{1}{x} \sum_{d \leq x^{u}} f(d) \sum_{m \leq x / d} \frac{1}{T(m d)} \\
& =\frac{F(\alpha, I)}{\Gamma(\alpha)} \sum_{d \leq x^{u}} \frac{f(d) g^{*}(d)}{d \log ^{\beta}(x / d)}+\mathrm{O}\left(\sum_{d \leq x^{u}} \frac{f(d) r(d)}{d \log ^{\beta+1}(x / d)}\right) \\
& =: \frac{F(\alpha, I)}{\Gamma(\alpha)} \sum_{d \leq x^{u}} \frac{f(d) g^{*}(d)}{d \log ^{\beta}(x / d)}+\widehat{R}_{x}(u) .
\end{aligned}
$$

In the same region, summing by parts and using (6) we obtain

$$
\widehat{R}_{x}(u) \ll \frac{1}{\log ^{\beta+1} x}\left(\frac{1}{(u \log x)^{\alpha}}+\int_{1}^{x^{u}} \sum_{d \leq v} f(d) r(d) \frac{d v}{v^{2}}\right) \ll \frac{1}{u^{\alpha} \log x} .
$$


Hence

$$
\begin{aligned}
\frac{\Gamma(\alpha)}{F(\alpha, I)} \widehat{S}_{x}(u)= & \frac{1}{((1-u) \log x)^{\beta}} \frac{1}{x^{u}} \sum_{d \leq x^{u}} f(d) g^{*}(d)+\mathrm{O}\left(\frac{1}{u^{\alpha} \log x}\right) \\
& +\int_{1} \sum_{d \leq v} f(d) g^{*}(d)\left(\frac{1}{v^{2} \log ^{\beta}(x / v)}+\frac{\beta}{v^{2} \log ^{\beta+1}(x / v)}\right) d v \\
= & \int_{1}^{x^{u}} \sum_{d \leq v} f(d) g^{*}(d) \frac{d v}{v^{2} \log ^{\beta}(x / v)}+\mathrm{O}\left(\frac{1}{u^{\alpha} \log x}\right) .
\end{aligned}
$$

Further applying (10) and (12) we have

$$
\begin{aligned}
S_{x}(u, 1 / 2)= & \frac{1}{\Gamma(\alpha) \Gamma(\beta)} \int_{x^{u}}^{x^{1 / 2}} \frac{d v}{v\left(\log ^{\alpha} v\right) \log ^{\beta}(x / v)} \\
& +\mathrm{O}\left(\frac{1}{u^{\alpha} \log x}\right) .
\end{aligned}
$$

Substituting $v=x^{t}$, we obtain the desired formula for $1 / \log x \leq u \leq 1 / 2$.

(B) Now let $1 / 2 \leq v \leq 1-1 / \log x$ and

$$
\begin{aligned}
S_{x}(v) & =\frac{1}{x} \sum_{n \leq x} \frac{T\left(n, n^{v}\right)}{T(m)} \\
& =1-\frac{1}{x} \sum_{n \leq x} \frac{1}{T(n)} \sum_{\substack{d \mid n \\
d>x^{v}}} f(d)-\frac{1}{x} \sum_{n \leq x} \frac{1}{T(n)} \sum_{\substack{d \mid n \\
n^{v}<d \leq x^{v}}} f(d) \\
& =: 1-\check{S}_{x}(v)-\check{R}_{x}(v) .
\end{aligned}
$$

Set

$$
d(v)=e^{\beta v /(1-v)} \leq e^{-\beta} x^{\beta}
$$

Then by Lemma 4 ,

$$
\begin{aligned}
\check{R}_{x}(u) & =\frac{1}{x}\left(\sum_{d \leq d(v)}+\sum_{d(v)<d \leq x^{v}}\right) f(d) \sum_{m \leq d^{(1-v) / v}} \frac{1}{T(m d)} \\
\ll & \frac{1}{x} \sum_{d \leq d(v)} \frac{f(d)}{T(d)} \\
& +\frac{1}{x} \sum_{d(v)<d \leq x^{v}} f(d) \frac{d^{(1-v) / v}}{\left(\log d^{(1-v) / v)^{\beta}}\right.}\left(g^{*}(d)+\frac{r(d)}{(1-v) \log d}\right) .
\end{aligned}
$$


The first sum can be estimated using Lemma 2. Checking that the function $t / \log ^{\beta} t$ is increasing for $t \geq e^{\beta}$, we obtain

$$
\begin{aligned}
\check{R}_{x}(u) \ll & \frac{1}{x} \frac{d(v)}{\log d(v)} \exp \left\{\sum_{p \leq d(v)} \frac{f(p)}{p(1+f(p))}\right\} \\
& +\frac{1}{((1-v) \log x)^{\beta}} \frac{1}{x^{v}} \sum_{d \leq x^{v}} f(d)\left(g^{*}(d)+r(d)\right) \\
& \ll \frac{1}{x^{\alpha}}+\frac{1}{((1-v) \log x)^{\beta} \log ^{\alpha} x} \ll \frac{1}{(1-v)^{\beta} \log x} .
\end{aligned}
$$

Here we have also used Lemmas 3 and 4 .

Dealing with $\breve{S}_{x}(v)$ we replace $d$ by $n / d$. Then applying Lemma 3 we obtain

$$
\begin{aligned}
\check{S}_{x}(v)= & \frac{1}{x} \sum_{d \leq x^{1-v}} \sum_{x^{v}<m \leq x / d} \frac{f(m)}{T(m d)} \\
= & \frac{F(\beta ; f)}{\Gamma(\beta)} \sum_{d \leq x^{1-v}} \frac{1}{d \log ^{\alpha}(x / d)}\left(g(d)+\mathrm{O}\left(\frac{r(d)}{\log (x / d)}\right)\right) \\
& -\frac{F(\beta ; f)}{\Gamma(\beta) x^{1-v} \log ^{\alpha} x^{v}} \sum_{d \leq x^{1-v}}\left(g(d)+\mathrm{O}\left(\frac{r(d)}{\log x}\right)\right) \\
= & -\frac{F(\beta ; f)}{\Gamma(\beta)} \int_{1}^{x^{1-v}} \sum_{d \leq y} g(d) d\left(\frac{1}{y \log ^{\alpha}(x / y)}\right) \\
& +\left(\sum_{d \leq x^{1-v}} \frac{r(d)}{d}+\frac{1}{x^{1-v}} \sum_{d \leq x^{1-v}} r(d)\right) \mathrm{O}\left(\frac{1}{\log ^{\alpha+1} x}\right) .
\end{aligned}
$$

By Lemmas 2 and 3, the terms containing the function $r(d)$ contribute the error $\mathrm{O}\left((1-v)^{-\beta} \log ^{-1} x\right)$, thus, by (13) and Lemma 3 again,

$$
\begin{aligned}
S_{x}(1 / 2, v)= & \check{S}_{x}(1 / 2)-\check{S}_{x}(v)+\mathrm{O}\left((1-v)^{-\beta} \log ^{-1} x\right) \\
= & \frac{1}{\Gamma(\alpha) \Gamma(\beta)} \int_{x^{1-v}}^{x^{1 / 2}}\left(1+\mathrm{O}\left(\frac{1}{\log y}\right)\right) \frac{d y}{y \log ^{\beta} y \log ^{\alpha}(x / y)} \\
& +\mathrm{O}\left(\int_{x^{1-v}}^{x^{1 / 2}} \frac{d y}{y \log ^{\beta} y \log ^{\alpha+1}(x / y)}\right)+\mathrm{O}\left(\frac{1}{(1-v)^{\beta} \log x}\right) .
\end{aligned}
$$

Substituting $y=x^{t}$ and recalling our notation, we obtain

$$
\begin{aligned}
S_{x}(1 / 2, v) & =B(1-v, 1 / 2 ; \beta, \alpha)+\mathrm{O}\left((1-v)^{-\beta} \log ^{-1} x\right) \\
& =B(1 / 2, v ; \alpha, \beta)+\mathrm{O}\left((1-v)^{-\beta} \log ^{-1} x\right) .
\end{aligned}
$$

Theorem 2 is proved. 
5. Proof of Corollary. We will use the properties of the Dirichlet $L(s, \chi)$ functions.

Lemma 5 (see [8, Theorems 4.2, 5.7, and 5.8]). Fix $m \in \mathbb{N}$ and let $\chi$ be an arbitrary Dirichlet character modulo $m$. If $c_{3}=c_{3}(m)>0$ is sufficiently small, in the region $\sigma \geq 1-c_{3} / \log (|t|+2), t \in \mathbb{R}$, we have $L(s, \chi) \neq 0$ and

$$
L(s, \chi) \ll \log (|t|+2) .
$$

Proof of Corollary. It suffices to show that the function $f(m)$ defined via $a_{l}, 1 \leq l<m$ and $(l, m)=1$, belongs to the class $\mathcal{M}(\alpha)$, where $\alpha$ is as in the Corollary. By classical calculations (see [8, Section 4.3]) we explore the sum

$$
P(s):=\sum_{p}\left(\frac{1}{1+f(p)}-\alpha\right) \frac{1}{p^{s}}=\sum_{\substack{1 \leq l<m \\(l, m)=1}}\left(\frac{1}{1+a_{l}}-\alpha\right) \sum_{p \equiv l(\bmod m)} \frac{1}{p^{s}}+H_{4}(s),
$$

where $H_{4}(s)$ is an entire function corresponding to $p \mid m$. In the region defined in Lemma 5 , we can use the main branch of $\log L(s, \chi)$ defined by $L(\sigma, \chi) \rightarrow 0$ as $\sigma \rightarrow \infty$. For $\sigma>1$, we have

$$
\log L(s, \chi)=-\sum_{p} \log \left(1-\frac{\chi(p)}{p^{s}}\right)^{-1}=\sum_{p} \frac{\chi(p)}{p^{s}}+H_{5}(s, \chi),
$$

with $H_{5}(s, \chi)$ holomorphic in $\sigma>1 / 2$ and bounded in $\sigma \geq 3 / 4$. By the orthogonality of characters,

$$
\begin{aligned}
\sum_{p \equiv l(\bmod m)} \frac{1}{p^{s}} & =\frac{1}{\varphi(m)} \sum_{\chi} \overline{\chi(l)} \sum_{p} \frac{\chi(p)}{p^{s}} \\
& =\frac{1}{\varphi(m)} \sum_{\chi} \overline{\chi(l)}\left(\log L(s, \chi)+H_{5}(s, \chi)\right) .
\end{aligned}
$$

Hence

$$
\begin{aligned}
& P(s)=\frac{1}{\varphi(m)} \sum_{\substack{1 \leq l<m \\
(l, m)=1}}\left(\frac{1}{1+a_{l}}-\alpha\right) \sum_{\chi} \overline{\chi(l)} \log L(s, \chi)+H_{6}(s) \\
& =\frac{1}{\varphi(m)} \sum_{\substack{1 \leq l<m \\
(l, m)=1}}\left(\frac{1}{1+a_{l}}-\alpha\right) \sum_{\chi \neq \chi_{0}} \overline{\chi(l)} \log L(s, \chi)+H_{6}(s)
\end{aligned}
$$

with some $H_{6}(s)$ holomorphic and bounded in $\sigma \geq 3 / 4$. In the last step we exploited the choice of $\alpha$ and set $\chi_{0}$ to be the principal character. By Lemma 5 the last equality gives the desired analytic continuation of $P(s)$ into the region $\sigma \geq 1-c_{3} / \log (2+|t|)$ where $P(s)$ is holomorphic. Moreover, by Lemma 5 , in the same region we obtain $P(s) \ll \log \log (3+|t|)$, where the constant in $\ll$ depends also on $m$ and the constants $a_{l}$. 
Consequently, $f(m) \in \mathcal{M}(\alpha)$ and the assertion of the Corollary follow from the theorems.

\section{References}

[1] G. Bareikis, Beta distribution in the polynomial semigroup, Ann. Univ. Sci. Budapest. Sect. Comp. 22 (2003), 35-48.

[2] J.-M. Deshouillers, F. Dress et G. Tenenbaum, Lois de répartition des diviseurs, I, Acta Arith. 34 (1979), 273-285.

[3] F. Dress, Le théorème DDT, in: Séminaire de Théorie des Nombres 1977-1978, Exp. no. 8, CNRS, Talence, 1978, 9 pp.

[4] H. Halberstam and H. E. Richert, On a result of R. R. Hall, J. Number Theory 11 (1979), 76-89.

[5] E. Manstavičius, Natural divisors and the brownian motion, J. Théor. Nombres Bordeaux 8 (1996), 159-171.

[6] - Functional limit theorems in probabilistic number theory, in: Paul Erdős and his Mathematics, Bolyai Soc. Math. Stud. 11, G. Halász et al. (eds.), 2002, 465-491.

[7] E. Manstavičius and N. M. Timofeev, A functional limit theorem related to natural divisors, Acta Math. Hungar. 75 (1997), 1-13.

[8] E. Prachar, Primzahlverteilung, Grundlehren Math. Wiss. 91, Springer, 1957.

[9] G. Tenenbaum, Lois de répartition des diviseurs, IV, Ann. Inst. Fourier (Grenoble) 29 (1979), no. 3, 1-15.

[10] - Introduction to Analytic and Probabilistic Number Theory, Cambridge Univ. Press, 1995.

[11] —, Addendum to the paper of E. Manstavičius and N. M. Timofeev "A functional limit theorem related to natural divisors", Acta Math. Hungar. 75 (1997), 15-22.

Gintautas Bareikis

Department of Mathematics and Informatics

Vilnius University

Naugarduko St. 24

LT-03225 Vilnius, Lithuania

E-mail: gintautas.bareikis@maf.vu.lt
Eugenijus Manstavičius

Institute of Mathematics and Informatics

Akademijos St. 4

LT-08663 Vilnius, Lithuania

E-mail: eugenijus.manstavicius@maf.vu.lt 